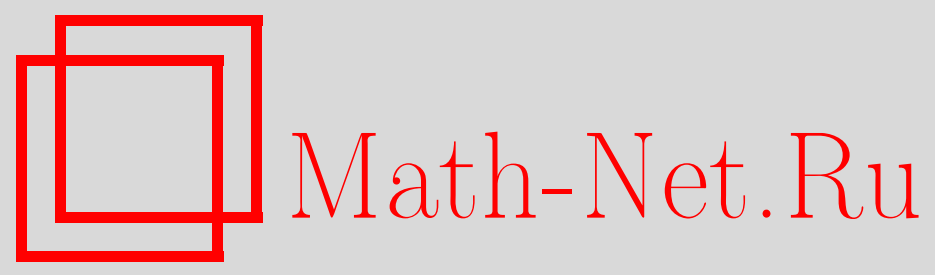

А. С. Рыбаков, Модификация алгоритма оценки количества целых чисел, имеющих не более трех болыших простых делителей, Матем. вопр. криптогр., 2013, том 4, выпуск 3, 131-158

DOI: https://doi.org/10.4213/mvk95

Использование Общероссийского математического портала Math-Net.Ru подразумевает, что вы прочитали и согласны с пользовательским соглашением

http://www.mathnet.ru/rus/agreement

Параметры загрузки:

IP : 54.224 .135 .184

26 апреля 2023 г., $11: 51: 52$ 
УДК: 511.333

\title{
Модификация алгоритма оценки количества целых чисел, имеющих не более трех больших простых делителей
}

\author{
А. С. Рыбаков \\ Лаборатория ТВП, Москва
}

Получено 20.IV.2012

В работе предлагаются методы быстрого вычисления некоторых обобщений функции Дикмана, позволяющих оценивать количество целых чисел в интервале, имеющих не более трех больших простых делителей.

Ключевые слова: гладкие и полугладкие числа, функция Дикмана, интегральные представления, быстро сходящиеся разложения

A modification of an algorithm for estimating the cardinality of integers with no more than three prime divisors

\section{A. S. Rybakov}

TPA Laboratory, Moscow

Abstract. We suggest fast computational methods for some generalizations of the Dickman function used to estimate the cardinality of the set of integers in an interval having no more than three prime divisors.

Key words: smooth and semi-smooth numbers, Dickman function, integral representations, quickly convergent series

Citation: Mathematical Aspects of Cryptography, 2013, vol. 4, no. 3, pp. 131-158 (Russian). 


\section{1. Введение}

В данной работе речь пойдет о разработке быстрых алгоритмов вычисления некоторых обобщений хорошо известной функции Дикмана $\rho(u)$, т. е. решения дифференциального уравнения $u \rho^{\prime}(u)+\rho(u-1)=0$ с начальными условиями $\rho(u)=0$ при $u<0, \rho(u)=1$ при $0 \leqslant u \leqslant 1$, см., например, [5]. Более точно, речь будет идти о функциях $G_{m}\left(\alpha, \beta_{1}, \beta_{2}\right), m=1,2, \ldots$, равных по определению предельной при $\alpha \rightarrow \infty$ вероятности того, что все простые делители случайно равновероятно выбранного целого числа из отрезка $[1, x]$ не превосходят $x^{\alpha}$, за исключением точно $m$ простых делителей $q$ (с учетом кратностей), лежащих в промежутке $\left(x^{\beta_{1}}, x^{\beta_{2}}\right]$. Такие числа обычно называются строго $\left(x^{\alpha}, x^{\beta_{1}}, x^{\beta_{2}}, m\right)$-полугладкими.

Вопросы быстрого вычисления функций $G_{m}$ с заданной точностью при некоторых значениях $m$ исследовались в ряде работ. В частности, для случая $m=0,1$ и $\alpha=\beta_{1}$ быстрый алгоритм нахождения значений $G_{m}$ предложен в работе Э. Баха и Р. Перальты [3], являющейся по существу исходной точкой развития всех дальнейших методов, касающихся функций более сложного вида. Случай $m=2, \alpha=\beta_{1}$ разобран в работе Р. Ламберта [6], где показано, что при $K=\left\lceil\frac{\beta_{2}}{\alpha}\right\rceil+2$ нахождение величины $G_{2}\left(\alpha, \alpha, \beta_{2}\right)$ требует $O\left(K \ln ^{2} K\right)$ операций сложения и умножения с плавающей точкой, $O(K)$ операций деления и $O(K)$ операций нахождения значений логарифма и дилогарифма, где $\operatorname{dilog}(x)=\int_{1}^{x} \frac{\ln t}{1-t} d t$.

В настоящей работе предлагается более быстрый алгоритм оценки величины $G_{2}\left(\alpha, \beta_{1}, \beta_{2}\right)$, требующий лишь $O(K \ln K)$ операций сложения и умножения с плавающей точкой, $O(K)$ операций деления и $O(K)$ операций нахождения значений логарифма. Подчеркнем, что никаких других операций, кроме названных, в нашем методе не используется. В частности, значения дилогарифма вычислять не нужно. Наш метод работает и в более общей ситуации, когда число $\beta_{1}$ может быть не равно $\alpha$.

Отметим, что для случая $m=2$ с произвольным $\beta_{1}$ в статье В.Х.Эккелькамп [4] предложен алгоритм, обобщающий алгоритм из упомянутой выше работы Ламберта. При этом сохраняет место относительная неэффективность данного алгоритма, выражающаяся в чрезмерном количестве арифметических операций.

Случай $m=3$ существенно труднее, чем случаи $m=0,1,2$, поскольку с ростом $m$ растет и кратность интеграла в формуле вычисления величины $G_{m}$. При $m=3$ надо брать уже тройной интеграл, и задача разработки алгоритма, аналогичного исходному алгоритму Э. Баха и Р. Перальты, является намного более сложной. Основным результатом настоящей работы является 
построение такого алгоритма. Как и в случае $m=2$, он требует $O(K \ln K)$ операций сложения и умножения с плавающей точкой, $O(K)$ операций деления и $O(K)$ операций нахождения значений логарифма.

Другим основным результатом настоящей работы является доказательство следующей теоремы.

Теорема 1. Пусть $G_{m}\left(\alpha, \beta_{1}, \beta_{2}\right)-$ предельная при $x \rightarrow \infty$ плотность множества чисел отрезка $[1, x]$ при $x \rightarrow \infty$, все простые делители которых не превосходят $x^{\alpha}$, за исключением точно $m$ простых делителей q (с учетом кратностей), лежащих в промежутке $\left(x^{\beta_{1}}, x^{\beta_{2}}\right]$. Тогда имеют место равенства

$$
G_{2}\left(\alpha, \beta_{1}, \beta_{2}\right)=\sum_{w \in\left\{\beta_{1}, \beta_{2}\right\}} e(w) \int_{\beta_{1}+w}^{\beta_{2}+w} \rho\left(\frac{1-\mu_{1}}{\alpha}\right) \frac{d \mu_{1}}{\mu_{1}} \cdot \ln \frac{\mu_{1}-w}{w}
$$

$u$

$$
\begin{gathered}
G_{3}\left(\alpha, \beta_{1}, \beta_{2}\right)=\frac{1}{3} \sum_{w_{1}, w_{2} \in\left\{\beta_{1}, \beta_{2}\right\}} e\left(w_{1}\right) e\left(w_{2}\right) \times \\
\times \int_{\beta_{1}+w_{1}+w_{2}}^{\beta_{2}+w_{1}+w_{2}} \rho\left(\frac{1-\mu_{1}}{\alpha}\right) d \mu_{1} \int_{w_{1}+w_{2}}^{\beta_{1}} \frac{d \mu_{2}}{\left(\mu_{1}-\mu_{2}\right) \mu_{2}} \cdot \ln \frac{\mu_{2}-w_{2}}{w_{2}},
\end{gathered}
$$

где $\rho-$ функиия Дикмана, е $(๗)=1$, если

В работе приведены конкретные алгоритмы, которые позволяют эффективно вычислять значения сумм интегралов, представленных формулами (1) и (2).

В заключительной части работы приводится ряд численных результатов, полученных в ходе реализации предложенных алгоритмов на языке С. В частности, приводится ряд таблиц с численными данными, показывающих практические возможности этих алгоритмов по сравнению с некоторыми известными способами оценки величин $G_{m}, m=2,3$, с помощью квадратурных формул.

\section{2. Интегральные формулы для функций $\boldsymbol{G}_{2}$ и $\boldsymbol{G}_{3}$}

Обозначим через $\Psi_{m}\left(x, x^{\alpha}, x^{\beta_{1}}, x^{\beta_{2}}\right)$ вероятность того, что все простые делители случайно равновероятно выбранного целого числа $n$ отрезка $[1 ; x]$ 
не превосходят $x^{\alpha}$, за исключением точно $m$ простых делителей $q$ (с учетом кратностей), лежащих в промежутке $\left(x^{\beta_{1}}, x^{\beta_{2}}\right]$. В дальнейшем такие числа $n$ будем для краткости называть строго $\left(x^{\alpha}, x^{\beta_{1}}, x^{\beta_{2}}, m\right)$-полугладкими. Если же число $n$ имеет лишь не более $m$ простых делителей $q$ (с учетом кратностей), лежащих в указанном промежутке, при сохранении тех же условий относительно малых делителей, то данное число будем называть $\left(x^{\alpha}, x^{\beta_{1}}, x^{\beta_{2}}, m\right)$-полугладким.

Пусть $G_{m}\left(\alpha, \beta_{1}, \beta_{2}\right)$ - предел отношения $\Psi_{m}\left(x, x^{\alpha}, x^{\beta_{1}}, x^{\beta_{2}}\right) / x$ при $x \rightarrow$ $\rightarrow \infty$. В данном разделе нас будут интересовать явные формулы для функций $G_{m}$ в двух частных случаях: $m=2$ и $m=3$. Для вывода этих формул удобно обозначить через $n_{(i)}$ число, равное $i$-му по порядку элементу отсортированного по невозрастанию набора простых делителей натурального числа $n$, если в этом наборе имеется не менее $i$ элементов с учетом кратностей, и 1 в остальных случаях.

Тогда по определению

$$
\Psi_{2}\left(x, x^{\alpha}, x^{\beta_{1}}, x^{\beta_{2}}\right)=\sum_{x^{\beta_{1}}<p_{1} \leq x^{\beta_{2}}} \sum_{x^{\beta_{1}}<p_{2} \leq p_{1}} \#\left\{n \leq x: n_{(1)}=p_{1}, n_{(2)}=p_{2}, n_{(3)} \leq x^{\alpha}\right\}
$$

и

$$
\begin{aligned}
& \Psi_{3}\left(x, x^{\alpha}, x^{\beta_{1}}, x^{\beta_{2}}\right)= \sum_{x^{\beta_{1}}<p_{1} \leq x^{\beta_{2}}} \sum_{x^{\beta_{1}}<p_{2} \leq p_{1}} \sum_{x^{\beta_{1}}<p_{3} \leq p_{2}} \#\{n \leq x: \\
&\left.n_{(1)}=p_{1}, n_{(2)}=p_{2}, n_{(3)}=p_{3}, n_{(4)} \leq x^{\alpha}\right\} .
\end{aligned}
$$

Будем предполагать, что $\beta_{2}<1 / 2$ при $m=2$ и $\beta_{2}<1 / 3$ при $m=3$. Заметим, что для практики эти условия не являются ограничительными: во всех известных рекордах факторизации они имеют место. Тогда в написанных выше суммах произведение чисел $p_{1}, \ldots, p_{m}$ будет всегда меньше $x$. Применяя это к формулам для функций $\Psi_{2}$ и $\Psi_{3}$, получим

$$
\begin{aligned}
& \Psi_{2}\left(x, x^{\alpha}, x^{\beta_{1}}, x^{\beta_{2}}\right)=\sum_{x^{\beta_{1}}<p_{1} \leq x^{\beta_{2}}} \sum_{x^{\beta_{1}}<p_{2} \leq p_{1}} \#\left\{k \leq \frac{x}{p_{1} p_{2}}: k_{(1)} \leq x^{\alpha}\right\}= \\
& =\sum_{x^{\beta_{1}}<p_{1} \leq x^{\beta_{2}}} \sum_{x^{\beta_{1}}<p_{2} \leq p_{1}} \#\left\{k \leq \frac{x}{p_{1} p_{2}}: k_{(1)} \leq\left(\frac{x}{p_{1} p_{2}}\right)^{\frac{\alpha}{1-\ln \left(p_{1} p_{2}\right) / \ln x}}\right\}
\end{aligned}
$$


и

$$
\begin{aligned}
& \Psi_{3}\left(x, x^{\alpha}, x^{\beta_{1}}, x^{\beta_{2}}\right)=\sum_{x^{\beta_{1}}<p_{1} \leq x^{\beta_{2}}} \sum_{x^{\beta_{1}}<p_{2} \leq p_{1}} \sum_{x^{\beta_{1}<p_{3} \leq p_{2}}} \#\left\{k \leq \frac{x}{p_{1} p_{2} p_{3}}: k_{(1)} \leq x^{\alpha}\right\}= \\
& =\sum_{x^{\beta_{1}}<p_{1} \leq x^{\beta_{2}}} \sum_{x^{\beta_{1}<p_{2} \leq p_{1}}} \sum_{x^{\beta_{1}}<p_{3} \leq p_{2}} \#\left\{k \leq \frac{x}{p_{1} p_{2} p_{3}}: k_{(1)} \leq\left(\frac{x}{p_{1} p_{2} p_{3}}\right)^{\frac{\alpha}{1-\ln \left(p_{1} p_{2} p_{3}\right) / \ln x}}\right\} .
\end{aligned}
$$

Пользуясь свойствами функции Дикмана $\rho(x)$ (см., например, [3]), эти равенства можно переписать в виде

$$
\Psi_{2}\left(x, x^{\alpha}, x^{\beta_{1}}, x^{\beta_{2}}\right)=(1+o(1)) \sum_{x^{\beta_{1}}<p_{1} \leq x^{\beta_{2}}} \sum_{x^{\beta_{1}}<p_{2} \leq p_{1}} \frac{x}{p_{1} p_{2}} \cdot \rho\left(\frac{1-\ln \left(p_{1} p_{2}\right) / \ln x}{\alpha}\right)
$$

и

$$
\begin{aligned}
& \Psi_{3}\left(x, x^{\alpha}, x^{\beta_{1}}, x^{\beta_{2}}\right)= \\
& =(1+o(1)) \sum_{x^{\beta_{1}}<p_{1} \leq x^{\beta_{2}}} \sum_{x^{\beta_{1}}<p_{2} \leq p_{1}} \sum_{x^{\beta_{1}}<p_{3} \leq p_{2}} \frac{x}{p_{1} p_{2} p_{3}} \cdot \rho\left(\frac{1-\ln \left(p_{1} p_{2} p_{3}\right) / \ln x}{\alpha}\right) .
\end{aligned}
$$

Здесь буквы $p_{1}, p_{2}$ и $p_{3}$ обозначают простые числа. Поскольку количество простых чисел, не превосходящих $x$, асимптотически равно $x / \ln x$, то, используя преобразование Абеля, от сумм по простым числам можно перейти к суммам по произвольным натуральным числам:

$$
\begin{aligned}
& \Psi_{2}\left(x, x^{\alpha}, x^{\beta_{1}}, x^{\beta_{2}}\right)= \\
& =(1+o(1)) \sum_{x^{\beta_{1}}<k_{1} \leq x^{\beta_{2}}} \sum_{x^{\beta_{1}}<k_{2} \leq k_{1}} \frac{x}{k_{1} \ln k_{1} \cdot k_{2} \ln k_{2}} \cdot \rho\left(\frac{1-\ln \left(k_{1} k_{2}\right) / \ln x}{\alpha}\right)
\end{aligned}
$$

и

$$
\begin{aligned}
& \Psi_{3}\left(x, x^{\alpha}, x^{\beta_{1}}, x^{\beta_{2}}\right)= \\
& =(1+o(1)) \sum_{x^{\beta_{1}<k_{1} \leq x^{\beta_{2}}}} \sum_{x^{\beta_{1}}<_{2} \leq k_{1}} \sum_{x^{\beta_{1}<k_{3} \leq k_{2}}} \frac{x}{k_{1} \ln k_{1} \cdot k_{2} \ln k_{2} \cdot k_{3} \ln k_{3}} \times \\
& \times \rho\left(\frac{1-\ln \left(k_{1} k_{2} k_{3}\right) / \ln x}{\alpha}\right) .
\end{aligned}
$$

Заменяя кратные суммы интегралами, найдем

$$
\Psi_{2}\left(x, x^{\alpha}, x^{\beta_{1}}, x^{\beta_{2}}\right)=(1+o(1)) \cdot x \cdot \int_{x^{\beta_{1}}}^{x^{\beta_{2}}} \int_{x^{\beta_{1}}}^{t_{1}} \frac{d t_{1}}{t_{1} \ln t_{1}} \cdot \frac{d t_{2}}{t_{2} \ln t_{2}} \cdot \rho\left(\frac{1-\ln \left(t_{1} t_{2}\right) / \ln x}{\alpha}\right)
$$


и

$$
\begin{aligned}
& \Psi_{3}\left(x, x^{\alpha}, x^{\beta_{1}}, x^{\beta_{2}}\right)= \\
& =1+o(1)) \cdot x \cdot \int_{x^{\beta_{1}}}^{x_{x^{\beta_{1}}}} \int_{x^{\beta_{1}}}^{t_{1}} \int_{t_{1}}^{t_{2}} \frac{d t_{1}}{t_{1} t_{1}} \cdot \frac{d t_{2}}{t_{2} \ln t_{2}} \cdot \frac{d t_{3}}{t_{3} \ln t_{3}} \cdot \rho\left(\frac{1-\ln \left(t_{1} t_{2} t_{3}\right) / \ln x}{\alpha}\right) .
\end{aligned}
$$

Сделаем в интегралах замену переменной

$$
\lambda_{i}=\frac{\ln t_{i}}{\ln x}, \quad d \lambda_{i}=\frac{d t_{i}}{t_{i} \cdot \ln x}=\frac{\lambda_{i} d t_{i}}{t_{i} \cdot \ln t_{i}}, \quad \frac{d t_{i}}{t_{i} \cdot \ln t_{i}}=\frac{d \lambda_{i}}{\lambda_{i}}, \quad i=1,2,3 .
$$

Тогда получим

$$
\Psi_{2}\left(x, x^{\alpha}, x^{\beta_{1}}, x^{\beta_{2}}\right)=(1+o(1)) \cdot x \cdot \int_{\beta_{1}}^{\beta_{2}} \int_{\beta_{1}}^{\lambda_{1}} \frac{d \lambda_{1}}{\lambda_{1}} \cdot \frac{d \lambda_{2}}{\lambda_{2}} \cdot \rho\left(\frac{1-\lambda_{1}-\lambda_{2}}{\alpha}\right)
$$

и

$$
\Psi_{3}\left(x, x^{\alpha}, x^{\beta_{1}}, x^{\beta_{2}}\right)=(1+o(1)) \cdot x \cdot \int_{\beta_{1}}^{\beta_{2}} \int_{\beta_{1}}^{\lambda_{1}} \int_{\beta_{1}}^{\lambda_{2}} \frac{d \lambda_{1}}{\lambda_{1}} \cdot \frac{d \lambda_{2}}{\lambda_{2}} \cdot \frac{d \lambda_{3}}{\lambda_{3}} \cdot \rho\left(\frac{1-\lambda_{1}-\lambda_{2}-\lambda_{3}}{\alpha}\right) .
$$

Отсюда непосредственно по определению функций $G_{2}$ и $G_{3}$ находим

$$
G_{2}\left(\alpha, \beta_{1}, \beta_{2}\right)=\int_{\beta_{1}}^{\beta_{2}} \int_{\beta_{1}}^{\lambda_{1}} \frac{d \lambda_{1}}{\lambda_{1}} \cdot \frac{d \lambda_{2}}{\lambda_{2}} \cdot \rho\left(\frac{1-\lambda_{1}-\lambda_{2}}{\alpha}\right)
$$

и

$$
G_{3}\left(\alpha, \beta_{1}, \beta_{2}\right)=\int_{\beta_{1}}^{\beta_{2}} \int_{\beta_{1}}^{\lambda_{1}} \int_{\beta_{1}}^{\lambda_{2}} \frac{d \lambda_{1}}{\lambda_{1}} \cdot \frac{d \lambda_{2}}{\lambda_{2}} \cdot \frac{d \lambda_{3}}{\lambda_{3}} \cdot \rho\left(\frac{1-\lambda_{1}-\lambda_{2}-\lambda_{3}}{\alpha}\right) .
$$

Переписывая последние формулы в виде

$$
\begin{gathered}
G_{2}\left(\alpha, \beta_{1}, \beta_{2}\right)=\iiint_{\beta_{1} \leqslant \lambda_{2} \leqslant \lambda_{1} \leqslant \beta_{2}} \frac{d \lambda_{1}}{\lambda_{1}} \cdot \frac{d \lambda_{2}}{\lambda_{2}} \cdot \rho\left(\frac{1-\lambda_{1}-\lambda_{2}}{\alpha}\right), \\
G_{3}\left(\alpha, \beta_{1}, \beta_{2}\right)=\iiint_{\beta_{1} \leqslant \lambda_{3} \leqslant \lambda_{2} \leqslant \lambda_{1} \leqslant \beta_{2}} \frac{d \lambda_{1}}{\lambda_{1}} \cdot \frac{d \lambda_{2}}{\lambda_{2}} \cdot \frac{d \lambda_{3}}{\lambda_{3}} \cdot \rho\left(\frac{1-\lambda_{1}-\lambda_{2}-\lambda_{3}}{\alpha}\right)
\end{gathered}
$$


и замечая, что подынтегральные функции инвариантны относительно перестановок аргументов, получим

$$
G_{2}\left(\alpha, \beta_{1}, \beta_{2}\right)=\frac{1}{2 !} \cdot \int_{\beta_{1}}^{\beta_{2}} \int_{\beta_{1}}^{\beta_{2}} \frac{d \lambda_{1}}{\lambda_{1}} \cdot \frac{d \lambda_{2}}{\lambda_{2}} \cdot \rho\left(\frac{1-\lambda_{1}-\lambda_{2}}{\alpha}\right)
$$

и

$$
G_{3}\left(\alpha, \beta_{1}, \beta_{2}\right)=\frac{1}{3 !} \cdot \int_{\beta_{1}}^{\beta_{2}} \int_{\beta_{1}}^{\beta_{2}} \int_{\beta_{1}}^{\beta_{2}} \frac{d \lambda_{1}}{\lambda_{1}} \cdot \frac{d \lambda_{2}}{\lambda_{2}} \cdot \frac{d \lambda_{3}}{\lambda_{3}} \cdot \rho\left(\frac{1-\lambda_{1}-\lambda_{2}-\lambda_{3}}{\alpha}\right) .
$$

Аналогичным образом выводится, что

$$
G_{m}\left(\alpha, \beta_{1}, \beta_{2}\right)=\frac{1}{m !} \cdot \int_{\left[\beta_{1}, \beta_{2}\right]^{m}} \frac{d \lambda_{1}}{\lambda_{1}} \cdot \ldots \cdot \frac{d \lambda_{m}}{\lambda_{m}} \cdot \rho\left(\frac{1-\lambda_{1}-\ldots-\lambda_{m}}{\alpha}\right)
$$

для произвольного натурального числа $m$.

Интегралы (5) имеют весьма специальный вид, что позволяет разработать быстрые методы их вычисления. Мы будем основываться на идее разложения функции $\rho$ в быстро сходящийся ряд Тейлора. Как известно [3], функция Дикмана $\rho(x)$ имеет особенности в целых точках $k=0,1,2, \ldots$ На любом отрезке $[k-1, k]$ она совпадает с аналитической функцией $\rho_{k}(x)$, задаваемой рядом Тейлора

$$
\rho_{k}(k-\xi)=\sum_{i=0}^{\infty} c_{i}^{(k)} \cdot \xi^{i}, \quad 0 \leq \xi \leq 1
$$

с радиусом сходимости 1 .

Коэффициенты $c_{i}^{(k)}$ определяются по рекуррентным формулам, которые мы здесь не приводим, так как они не будут использоваться при описании метода.

Ниже мы увидим, что вычислительно эффективнее использовать ряды Тейлора для функций $\rho_{k}(x)$ в полуцелых точках, а не в целых, как это обычно делалось раньше. Поэтому займемся нахождением формул для коэффициентов такого разложения. 


\section{3. Модификация алгоритма вычисления функции Дикмана}

Ряд (6) не удобен тем, что область изменения аргумента $\xi$ в этом ряде имеет единицу в качестве правой граничной точки. Следствием этого является тот факт, что разности $v-w$ (где $v$ и $w-$ параметры интегралов раздела 5 ниже) могут равняться единице. В частности, степени $(v-w)^{i}$ могут не стремиться к нулю с ростом $i$, а это неудобно для вычислений. Это можно обойти разными путями, но проще всего разложить функцию $\rho_{k}$ в ряд не в целых, а в полуцелых точках:

$$
\rho_{k}(k-0.5-x)=\sum_{i \geq 0} c_{k, i} \cdot x^{i}, \quad-0.5 \leq x \leq 0.5, k=1,2, \ldots
$$

Сейчас мы докажем несколько простых утверждений, позволяющих вычислять коэффициенты $c_{k, i}$ разложения (7).

Напомним, что функция Дикмана определяется как решение дифференциально-разностного уравнения $u \rho^{\prime}(u)+\rho(u-1)=0$ с начальными условиями $\rho(x)=0$ для $x<0$ и $\rho(0)=1$. Прежде всего ясно, что $\rho_{1}(u)=1$, так что $c_{1,0}=1$ и $c_{1, i}=0$ при $i>0$.

Лемма 1. Справедливы равенства $c_{2,0}=1-\ln (3 / 2), c_{2, i}=(2 / 3)^{i} / i$ при $i>0$.

Доказательство. Так как $u \rho_{2}^{\prime}(u)+1=0$, то $\rho_{2}(u)=\rho_{1}(1)-\int_{1}^{u} \frac{d u}{u}=$ $1-\ln u$. Положив $u=3 / 2-x$, получим

$$
\rho_{2}(u)=1-\ln (3 / 2)-\ln \left(1-\frac{x}{3 / 2}\right)=1-\ln (3 / 2)+\sum_{i>0} \frac{(2 / 3)^{i}}{i} \cdot x^{i},
$$

откуда и следуют утверждения леммы.

Лемма 2. Справедливы равенства $c_{k, i}=\sum_{0 \leq j<i} c_{k-1, j} \cdot \frac{1}{i \cdot(k-0.5)^{i-j}}, i>0$, $k \geq 3$.

Доказательство. Так как $u \rho_{k}^{\prime}(u)+\rho_{k-1}(u-1)=0, k-2 \leq u \leq k$, то

$$
\rho_{k}(u)=\rho_{k}\left(u_{0}\right)-\int_{u_{0}}^{u} \frac{\rho_{k-1}(t-1)}{t} d t=\rho_{k}\left(u_{0}\right)+\int_{k-1 / 2-u_{0}}^{k-1 / 2-u} \frac{\rho_{k-1}(k-3 / 2-\xi)}{k-1 / 2-\xi} d \xi .
$$


Разлагая функцию $\rho_{k-1}$ в ряд и интегрируя почленно, получим

$\rho_{k}(u)=\rho_{k}\left(u_{0}\right)+\int_{x_{0}}^{x} \sum_{j \geq 0} c_{k-1, j} \cdot \frac{\xi^{j} d \xi}{k-1 / 2-\xi}=\rho_{k}\left(u_{0}\right)+\sum_{j \geq 0} c_{k-1, j} \cdot \int_{x_{0}}^{x} \frac{\xi^{j} d \xi}{k-1 / 2-\xi}$.

Обозначим последний интеграл через $A_{j}$. Эти интегралы можно вычислять рекуррентно:

$$
A_{j}=\int_{x_{0}}^{x} \frac{\xi^{j-1}(\xi-(k-1 / 2)+(k-1 / 2))}{k-\frac{1}{2}-\xi} d \xi=-\int_{x_{0}}^{x} \xi^{j-1} d \xi+(k-1 / 2) A_{j-1} .
$$

Отсюда по индукции получаем

$$
A_{j-1}=\left(\left.\frac{\xi^{j}}{j}\right|_{x_{0}} ^{x}+A_{j}\right) \frac{1}{k-1 / 2}=\left.\frac{\xi^{j}}{j}\right|_{x_{0}} ^{x} \cdot \frac{1}{k-1 / 2}+\left.\frac{\xi^{j+1}}{j+1}\right|_{x_{0}} ^{x} \cdot \frac{1}{(k-1 / 2)^{2}}+\ldots,
$$

T. e.

$$
A_{j}=\left.\sum_{i>j} \frac{\xi^{i}}{i}\right|_{x_{0}} ^{x} \cdot \frac{1}{(k-1 / 2)^{i-j}} .
$$

Подставляя эту формулу в (8), находим

$$
\begin{aligned}
\sum_{i>0} c_{k, i}\left(x^{i}-x_{0}^{i}\right) & =\left.\sum_{j \geq 0} c_{k-1, j} \sum_{i>j} \frac{\xi^{i}}{i}\right|_{x_{0}} ^{x} \cdot \frac{1}{(k-1 / 2)^{i-j}}= \\
& =\left.\sum_{i>0} \frac{\xi^{i}}{i}\right|_{x_{0}} ^{x} \sum_{0 \leq j<i} c_{k-1, j} \cdot \frac{1}{(k-1 / 2)^{i-j}},
\end{aligned}
$$

и наше утверждение доказано.

Лемма 3. При любом $a \geq 1$ имеем $\int_{a-1}^{a} \rho(u) d u=a \rho(a)$.

Доказательство. Так как $\rho(u)=-(u+1) \rho^{\prime}(u+1)$, то

$$
\begin{aligned}
\int_{a-1}^{a} \rho(u) d u & =-\int_{a-1}^{a}(u+1) \rho^{\prime}(u+1) d u=-\int_{a}^{a+1} u \rho^{\prime}(u) d u= \\
& =-\left.u \rho(u)\right|_{a} ^{a+1}+\int_{a}^{a+1} \rho(u) d u .
\end{aligned}
$$


Отсюда следует

$$
\begin{aligned}
& \int_{a-1}^{a} \rho(u) d u-a \rho(a)=\int_{a}^{a+1} \rho(u) d u-(a+1) \rho(a+1)= \\
& =\ldots=\int_{a+n-1}^{a+n} \rho(u) d u-(a+n) \rho(a+n),
\end{aligned}
$$

где $n$ - любое натуральное число. Переходя в последнем равенстве к пределу при $n \rightarrow \infty$ и используя известную асимптотику $\ln \rho(x) \sim-x \ln x, x \rightarrow \infty$ (см., например, [2]), получим требуемое.

Лемма 4. При любом целом $k \geq 2$

$$
(k-1) c_{k, 0}=0.5 c_{k-1,0}+\sum_{i>1} \frac{1}{i 2^{i}}\left(c_{k, i-1}-(-1)^{i} c_{k-1, i-1}\right) .
$$

Доказательство. Так как $(k-1 / 2) c_{k, 0}=(k-1 / 2) \rho(k-1 / 2)$, то по лемме 3

$$
\begin{aligned}
(k-1 / 2) c_{k, 0} & =\int_{k-3 / 2}^{k-1 / 2} \rho(u) d u=\int_{0}^{1} \rho(k-1 / 2-x) d x= \\
& =\int_{0}^{1 / 2} \rho_{k}(k-1 / 2-x) d x+\int_{1 / 2}^{1} \rho_{k-1}(k-1 / 2-x) d x= \\
& =\int_{0}^{1 / 2} \rho_{k}(k-1 / 2-x) d x+\int_{-1 / 2}^{0} \rho_{k-1}(k-3 / 2-x) d x= \\
& =\left.\sum_{i \geq 0} c_{k, i} \cdot \frac{x^{i+1}}{i+1}\right|_{0} ^{1 / 2}+\left.\sum_{i \geq 0} c_{k-1, i} \cdot \frac{x^{i+1}}{i+1}\right|_{-1 / 2} ^{0}= \\
& =\frac{1}{2}\left(c_{k, 0}+c_{k-1,0}\right)+\sum_{i>1} \frac{1}{i 2^{i}}\left(c_{k, i-1}-(-1)^{i} c_{k-1, i-1}\right) .
\end{aligned}
$$

Из последнего равенства непосредственно вытекает утверждение леммы. 
Лемма 5. При всех $i \geq 0 u k \geq 1$ имеют место неравенства $0 \leq c_{k, i} \leq 1.5^{-i}$

Доказательство. Заметим, что из дифференциально-разностного уравнения, определяющего функцию $\rho$, непосредственно следует, что эта функция монотонно убывает. Отсюда вытекает, что $c_{k, 0}=\rho(k-1 / 2) \leq \rho(0)=1$, т. е. при $i=0$ доказываемые неравенства выполнены. При $i>0$ и $k \leq 2$ в справедливости неравенств леммы убеждаемся непосредственной проверкой, пользуясь леммой 1 . При $i>0$ и $k \geq 3$ применим индукцию по $k$. Пользуясь леммой 2, получим

$0 \leq c_{k, i} \cdot 1.5^{i} \leq \sum_{0 \leq j<i} c_{k-1, j} \frac{1.5^{i}}{i \cdot(k-1 / 2)^{i-j}} \leq \sum_{0 \leq j<i} \frac{1.5^{i-j}}{i \cdot(k-1 / 2)^{i-j}} \leq \sum_{0 \leq j<i} \frac{1}{i}=1$,

и наше утверждение доказано.

В формулировках лемм 1, 2 и 4 приводятся формулы, позволяющие эффективно вычислять коэффициенты $c_{k, i}$ для всех значений индексов $k$ и $i$. Покажем, что при вычислении значений функции Дикмана по этим формулам достаточно ограничиться лишь индексами $i \leq 36$.

Согласно лемме 5 разность между $\rho_{k}\left(k-\frac{1}{2}-x\right)$ и начальным отрезком ряда Тейлора $\sum_{i \leq I} c_{k, i} x^{i}$ при $-\frac{1}{2} \leq x \leq \frac{1}{2}$ по модулю не превосходит $3^{-I}$. Для того, чтобы вычислить функцию $\rho_{k}\left(k-\frac{1}{2}-x\right),-\frac{1}{2} \leq x \leq \frac{1}{2}$ с ошибкой, меньшей $10^{-17}$ (точность, задаваемая разрядностью базового типа double), надо выбрать число $I$ так, что $3^{I}>10^{17}$, т. е. $I \geq 36$, и наше утверждение доказано.

Отметим, что во всех ранее предлагавшихся методах вычисления значений функций $G_{m}$ для функций $\rho_{k}(k-x)$ использовались ряды Тейлора (6) в окрестности точки 0 . Для коэффициентов $c_{i}^{(k)}$ этих рядов имеют место неравенства $\left|c_{i}^{(k)}\right|<2^{-i}$ при всех $i \geq 0$ и всех $k$ (см., например, [3]). Таким образом, для вычисления величины $\rho_{k}(k-x), 0 \leq x \leq 1$, с ошибкой, меньшей $10^{-17}$, с помощью начального отрезка ряда Тейлора $\sum_{i \leq I} c_{i}^{(k)} x^{i}$, надо взять число $I$ из условия $2^{I}>10^{17}$, т. е. $I \geq 56$. Сравнивая это значение с приведенным выше, видим, что размер оперативной памяти, отводимой под коэффициенты ряда Тейлора функции Дикмана, мы сократили в полтора раза без увеличения ошибки вычисления значений этой функции. 


\section{4. Модифицированные интегральные формулы для функций $\boldsymbol{G}_{2}$ и $\boldsymbol{G}_{3}$}

Возвращаясь к интегралам (5), сделаем унимодулярную замену переменной

$$
\mu_{i}=\lambda_{i}+\lambda_{i+1}+\ldots+\lambda_{m}, \quad i=1,2, \ldots, m,
$$

позволяющую упростить аргумент в функции $\rho$ под знаком интеграла и применить подготовленное выше разложение в ряд Тейлора. Тогда функция $G_{m}$ примет вид

$G_{m}\left(\alpha, \beta_{1}, \beta_{2}\right)=\frac{1}{m !} \cdot \int_{\beta_{1}}^{\beta_{2}} \frac{d \mu_{m}}{\mu_{m}} \cdot \int_{\beta_{1}+\mu_{m}}^{\beta_{2}+\mu_{m}} \frac{d \mu_{m-1}}{\mu_{m-1}-\mu_{m}} \cdot \ldots \cdot \int_{\beta_{1}+\mu_{2}}^{\beta_{2}+\mu_{2}} \frac{d \mu_{1}}{\mu_{1}-\mu_{2}} \cdot \rho\left(\frac{1-\mu_{1}}{\alpha}\right)$.

Основной прием, позволяющий ускорить вычисление значений функции $G_{m}$ с помощью формулы (9), состоит в многократном применении следующей элементарно доказываемой леммы, с помощью которой можно переставить аргументы и уменьшить кратность интегрирования.

Лемма 6. Пусть $\alpha, \beta, u_{0}, u_{1}$ - вещественные числа, $\phi(u, v)-$ интегрируемая функиия. Тогда, если $\alpha+u_{1}=\beta+u_{0}$, то

$$
\int_{u_{0}}^{u_{1}} d u \cdot \int_{\alpha+u}^{\beta+u} d v \cdot \phi(u, v)=\int_{\alpha+u_{0}}^{\alpha+u_{1}} d v \cdot \int_{u_{0}}^{v-\alpha} d u \cdot \phi(u, v)+\int_{\beta+u_{0}}^{\beta+u_{1}} d v \cdot \int_{v-\beta}^{u_{1}} d u \cdot \phi(u, v) .
$$

Применяя эту лемму непосредственно к интегралу $G_{2}$, получим

$$
\begin{gathered}
G_{2}\left(\alpha, \beta_{1}, \beta_{2}\right)=\frac{1}{2 !} \int_{\beta_{1}}^{\beta_{2}} \frac{d \mu_{2}}{\mu_{2}} \cdot \int_{\beta_{1}+\mu_{2}}^{\beta_{2}+\mu_{2}} \frac{d \mu_{1}}{\mu_{1}-\mu_{2}} \cdot \rho\left(\frac{1-\mu_{1}}{\alpha}\right)= \\
=\frac{1}{2 !} \int_{2 \beta_{1}}^{\beta_{1}+\beta_{2}} \rho\left(\frac{1-\mu_{1}}{\alpha}\right) d \mu_{1} \cdot \int_{\beta_{1}}^{\mu_{1}-\beta_{1}} \frac{d \mu_{2}}{\left(\mu_{1}-\mu_{2}\right) \mu_{2}}+ \\
+\frac{1}{2 !} \int_{\beta_{1}+\beta_{2}}^{2 \beta_{2}} \rho\left(\frac{1-\mu_{1}}{\alpha}\right) d \mu_{1} \cdot \int_{\mu_{1}-\beta_{2}}^{\beta_{2}} \frac{d \mu_{2}}{\left(\mu_{1}-\mu_{2}\right) \mu_{2}} .
\end{gathered}
$$


Последнюю формулу можно записать короче:

$$
G_{2}\left(\alpha, \beta_{1}, \beta_{2}\right)=\frac{1}{2 !} \sum_{w \in\left\{\beta_{1}, \beta_{2}\right\}} e(w) \int_{\beta_{1}+w}^{\beta_{2}+w} \rho\left(\frac{1-\mu_{1}}{\alpha}\right) d \mu_{1} \cdot \int_{w}^{\mu_{1}-w} \frac{d \mu_{2}}{\left(\mu_{1}-\mu_{2}\right) \mu_{2}}
$$

где $e(w)=1$, если $๗=\beta_{1}$ и $e(w)=-1$, если $๗=\beta_{2}$.

Чтобы использовать этот прием при преобразовании интеграла $G_{3}$, рассмотрим сначала интеграл несколько более общего вида, чем $G_{2}$ :

$$
G_{2}\left(\alpha, \beta_{1}, \beta_{2}, u_{1}, u_{2}\right)=\frac{1}{2 !} \int_{\beta_{1}+u_{1}}^{\beta_{2}+u_{1}} \frac{d \mu_{2}}{\mu_{2}} \cdot \int_{\beta_{1}+u_{2}+\mu_{2}}^{\beta_{2}+u_{2}+\mu_{2}} \frac{d \mu_{1}}{\mu_{1}-\mu_{2}} \cdot \rho\left(\frac{1-\mu_{1}}{\alpha}\right) .
$$

Рассуждая аналогично предыдущему, придем к формуле

$$
\begin{aligned}
& G_{2}\left(\alpha, \beta_{1}, \beta_{2}, u_{1}, u_{2}\right)= \\
& =\frac{1}{2 !} \sum_{w \in\left\{\beta_{1}, \beta_{2}\right\}} e(w) \int_{\beta_{1}+u_{1}+u_{2}+w}^{\beta_{2}+u_{1}+u_{2}+w} \rho\left(\frac{1-\mu_{1}}{\alpha}\right) d \mu_{1} \cdot \int_{u_{1}+w}^{\mu_{1}-u_{2}-w} \frac{d \mu_{2}}{\left(\mu_{1}-\mu_{2}\right) \mu_{2}} .
\end{aligned}
$$

Теперь все готово, чтобы преобразовать интеграл $G_{3}$ :

$$
\begin{gathered}
G_{3}\left(\alpha, \beta_{1}, \beta_{2}\right)=\frac{1}{3 !} \int_{\beta_{1}}^{\beta_{2}} \frac{d \mu_{3}}{\mu_{3}} \cdot \int_{\beta_{1}+\mu_{3}}^{\beta_{2}+\mu_{3}} \frac{d \mu_{2}}{\mu_{2}-\mu_{3}} \cdot \int_{\beta_{1}+\mu_{2}}^{\beta_{2}+\mu_{2}} \frac{d \mu_{1}}{\mu_{1}-\mu_{2}} \cdot \rho\left(\frac{1-\mu_{1}}{\alpha}\right)= \\
=\frac{1}{3 !} \sum_{w_{2} \in\left\{\beta_{1}, \beta_{2}\right\}} e\left(w_{2}\right) \int_{\beta_{1}+w_{2}}^{\beta_{2}+w_{2}} d \mu_{2} \cdot \int_{w_{2}}^{\mu_{2}-w_{2}} \frac{d \mu_{3}}{\left(\mu_{2}-\mu_{3}\right) \mu_{3}} \times \\
\times \int_{\beta_{1}+\mu_{2}}^{\beta_{2}+\mu_{2}} \frac{d \mu_{1}}{\mu_{1}-\mu_{2}} \cdot \rho\left(\frac{1-\mu_{1}}{\alpha}\right)=\frac{1}{3 !} \sum_{w_{2} \in\left\{\beta_{1}, \beta_{2}\right\}}^{\beta_{2}+w_{2}} e\left(w_{2}\right) \int_{\beta_{1}+w_{2}}^{\mu_{2}-w_{2}} d \mu_{2} \times \\
\times \int_{\beta_{1}+\mu_{2}}^{\beta_{2}+\mu_{2}} \frac{d \mu_{1}}{\mu_{1}-\mu_{2}} \cdot \rho\left(\frac{1-\mu_{1}}{\alpha}\right) \cdot \int_{w_{2}}^{d \mu_{3}} \frac{\left.\mu_{3}\right) \mu_{3}}{\left(\mu_{2}-\mu_{2}\right.}=
\end{gathered}
$$

2013, T. 4, № 3, C. 131-158 


$$
\begin{aligned}
=\frac{1}{3 !} & \sum_{w_{1}, w_{2} \in\left\{\beta_{1}, \beta_{2}\right\}} e\left(w_{1}\right) e\left(w_{2}\right) \int_{\beta_{1}+w_{1}+w_{2}}^{\beta_{2}+w_{1}+w_{2}} \rho\left(\frac{1-\mu_{1}}{\alpha}\right) d \mu_{1} \times \\
& \times \int_{w_{1}+w_{2}}^{\mu_{1}-w_{1}} \frac{d \mu_{2}}{\mu_{1}-\mu_{2}} \times \int_{w_{2}}^{\mu_{2}-w_{2}} \frac{d \mu_{3}}{\left(\mu_{2}-\mu_{3}\right) \mu_{3}} .
\end{aligned}
$$

В формулах (10) и (11) можно уменьшить кратность интегрирования, воспользовавшись тем, что

$$
\begin{gathered}
\int_{w}^{\mu_{2}-w} \frac{d \mu_{3}}{\left(\mu_{2}-\mu_{3}\right) \mu_{3}}=\frac{1}{\mu_{2}} \cdot \int_{w}^{\mu_{2}-w}\left(\frac{1}{\mu_{3}}+\frac{1}{\mu_{2}-\mu_{3}}\right) \cdot d \mu_{3}= \\
=\left.\frac{1}{\mu_{2}} \cdot \ln \frac{\mu_{3}}{\mu_{2}-\mu_{3}}\right|_{w} ^{\mu_{2}-w}=\frac{2}{\mu_{2}} \cdot \ln \frac{\mu_{2}-w}{\omega} .
\end{gathered}
$$

Таким образом, окончательно формулы для $G_{2}$ и $G_{3}$ принимают вид

$$
G_{2}\left(\alpha, \beta_{1}, \beta_{2}\right)=\sum_{w \in\left\{\beta_{1}, \beta_{2}\right\}} e(w) \int_{\beta_{1}+w}^{\beta_{2}+w} \rho\left(\frac{1-\mu_{1}}{\alpha}\right) \frac{d \mu_{1}}{\mu_{1}} \cdot \ln \frac{\mu_{1}-w}{w}
$$

и

$$
\begin{aligned}
G_{3}\left(\alpha, \beta_{1}, \beta_{2}\right)=\frac{1}{3} \sum_{w_{1}, w_{2} \in\left\{\beta_{1}, \beta_{2}\right\}} e\left(w_{1}\right) e\left(w_{2}\right) & \int_{\beta_{1}+w_{1}+w_{2}}^{\beta_{2}+w_{1}+w_{2}} \rho\left(\frac{1-\mu_{1}}{\alpha}\right) d \mu_{1} \times \\
& \times \int_{w_{1}+w_{2}}^{\mu_{1}-w_{1}} \frac{d \mu_{2}}{\left(\mu_{1}-\mu_{2}\right) \mu_{2}} \cdot \ln \frac{\mu_{2}-w_{2}}{w_{2}},
\end{aligned}
$$

т.е. совпадают с формулами, приведенными в теореме 1, и на этом доказательство теоремы заканчивается.

\section{5. Алгоритмы вычисления интегралов}

Вычисление интегралов (1) и (2) проводится в несколько этапов. Сначала мы опишем эффективный способ вычисления более простых интегралов:

(1) $J_{0}(u, v, w)=\int_{u}^{v}(t-w)^{i} d t$,

(2) $J_{1,1}\left(u, v, w, w_{1}\right)=\int_{u}^{v}(t-w)^{i} d t /\left(t-w_{1}\right)$,

(3) $J_{2,1}\left(u, v, w, w_{1}, w_{2}\right)=\int_{u}^{v}(t-w)^{i} \cdot \ln \left(t-w_{2}\right) d t /\left(t-w_{1}\right)$,

(4) $J_{2,2}\left(u, v, w, w_{2}\right)=\int_{u}^{v}(t-w)^{i} \cdot \ln \left(t-w_{2}\right) d t$,

(5) $J_{3,1}\left(u, v, w, w_{1}, w_{2}\right)=\int_{u}^{v}\left(t_{1}-w\right)^{i} d t_{1} \cdot \int_{w_{1}+w_{2}}^{t_{1}-w_{1}} d t_{2} \cdot \ln \left(t_{2}-w_{2}\right) /\left(t_{2}\left(t_{1}-t_{2}\right)\right)$, 
(6) $J_{3,2}\left(u, v, w, w_{1}, w_{2}\right)=\int_{u}^{v}\left(t_{1}-w\right)^{i} d t_{1} \cdot t_{1} \cdot \int_{w_{1}+w_{2}}^{t_{1}-w_{1}} d t_{2} \cdot \ln \left(t_{2}-w_{2}\right) /\left(t_{2}\left(t_{1}-t_{2}\right)\right)$,

(7) $F_{1}=\int_{A}^{B} \rho_{k}\left(\left(1-\mu_{1}\right) / \alpha\right) d \mu_{1} / \mu_{1}$,

(8) $F_{2}=\int_{A}^{B} \rho_{k}\left(\left(1-\mu_{1}\right) / \alpha\right) \cdot \ln \left(\left(\mu_{1}-\gamma_{1}\right) / \gamma_{1}\right) d \mu_{1} / \mu_{1}$,

(9) $F_{3}=\int_{A}^{B} \rho_{k}\left(\left(1-\mu_{1}\right) / \alpha\right) d \mu_{1} \int_{\gamma_{1}+\gamma_{2}}^{\mu_{1}-\gamma_{1}} \ln \left(\left(\mu_{2}-\gamma_{2}\right) / \gamma_{2}\right) d \mu_{2} /\left(\mu_{2}\left(\mu_{1}-\mu_{2}\right)\right)$.

В последних трех случаях предполагается, что $\left(1-\mu_{1}\right) / \alpha=k-0.5-x$, где $x \in[-0.5,0.5]$, если $\mu_{1} \in[A, B]$.

Интересующие нас функции $G_{2}$ и $G_{3}$ вычисляются через интегралы $F_{1}, F_{2}$ и $F_{3}$, а последние вычисляются через интегралы, обозначенные буквой $J$ с индексами. Поэтому мы начнем с описания алгоритмов быстрого вычисления интегралов типа $J$.

\section{1. Алгоритм вычисления значений интеграла $\boldsymbol{J}_{1,1}\left(\boldsymbol{u}, \boldsymbol{v}, \boldsymbol{w}, \boldsymbol{w}_{1}\right)$}

Интегралы

$$
J_{1,1}\left(u, v, w, w_{1}\right)=J_{1,1}[i]=\int_{u}^{v}(t-w)^{i} d t /\left(t-w_{1}\right)
$$

вычисляются по рекуррентной формуле

$$
J_{1,1}[i]=\left.\frac{(t-w)^{i}}{i}\right|_{u} ^{v}-\left(w-w_{1}\right) \cdot J_{1,1}[i-1], \quad i=1,2, \ldots
$$

Заметим, что величины $u-w$ и $v-w$ в дальнейшем всегда будут равны некоторому значению $x$ в разложении (7). Следовательно, можно считать, что $\max (|u-w|,|v-w|) \leq 0.5$.

Легко проверить, что для величины $x$, имеющей тип double и не превосходящей по модулю 0.5 , значение степени $x^{J}$ при $J \geq \operatorname{Jmax}=1075$ всегда равно нулю. Поэтому интеграл $J_{1,1}[i]$ равен нулю при $i \geq \operatorname{Jmax}$. Это на самом деле весьма важный момент, показывающий, что мы можем применять формулу (12) в обратном направлении, т. е. при $i=\mathrm{J} \max , J \max -1, \ldots, 1$. Вопрос о том, какое из направлений выбрать, прямое или обратное, целиком определяется значением величины $-w_{1}$. Если $\left|w-w_{1}\right|>1$, то мы выбираем обратное направление, предварительно переписав формулу (12) в виде

$$
J_{1,1}[i-1]=\left(\left.\frac{(t-w)^{i}}{i}\right|_{u} ^{v}-J_{1,1}[i]\right) /\left(w-w_{1}\right), \quad i=\mathrm{Jmax}, J \max -1, \ldots, 1 .
$$

При таком способе вычислений не будет происходить экспоненциального роста ошибки округления, как это было бы при прямом порядке. Если же $\left|w-w_{1}\right| \leq 1$, то надо применять непосредственно формулу (12), взяв $J_{1,1}[0]=\left.\ln \left(t-w_{1}\right)\right|_{u} ^{v}$. 


\section{2. Алгоритмы вычисления значений дилогарифмов}

В данном разделе мы покажем, как можно быстро вычислить следующие два интеграла:

(1) $\operatorname{DiLog} \mathrm{A}\left(u, v, w_{1}, w_{2}\right)=\int_{u}^{v} \ln \left(t-w_{2}\right) d t /\left(t-w_{1}\right), \max \left(w_{1}, w_{2}\right)<u<v$;

(2) $\operatorname{DiLogB}\left(u, v, w_{1}, w_{2}\right)=\int_{u}^{v} \ln \left(t-w_{2}\right) d t /\left(t-w_{1}\right), w_{2}<u<v<w_{1}$.

Как видно, подынтегральная функция здесь одна и та же, различными являются лишь области значений параметров на входе.

Отметим, что случаями (1) и (2) охватываются все возможные значения набора параметров дилогарифма. Действительно, не ограничивая общности, можно считать, что $u<v$. Кроме того, подынтегральная функция определена и интегрируема в том и только в том случае, когда $u>w_{2}$ и $w_{1} \notin[u, v]$. Первое из этих условий обеспечивает существование логарифма $\ln \left(t-w_{2}\right)$ на отрезке $[u, v]$, а второе условие - интегрируемость функции $1 /\left(t-w_{1}\right)$ на этом отрезке. Отсюда ясно, что точка $\max \left(w_{1}, w_{2}\right)$ не может лежать между точками $u$ и $v$. Если она лежит левее точек $u$ и $v$, то мы попадаем в случай (1). Если же она лежит правее точек $u$ и $v$, то она совпадает с $w_{1}$, и мы попадаем в случай (2).

Теперь покажем, как можно быстро вычислить значение дилогарифма в каждом из случаев (1) и (2).

Разберем для начала случай (1). Если $w_{2}>w_{1}$, то, пользуясь равенством

$$
\operatorname{DiLog} \mathrm{A}=\left.\ln \left(t-w_{1}\right) \cdot \ln \left(t-w_{2}\right)\right|_{u} ^{v}+I_{1}, \quad I_{1}=-\int_{u}^{v} \ln \left(t-w_{1}\right) d t /\left(t-w_{2}\right),
$$

сведем задачу вычисления дилогарифма к основному рассматриваемому ниже случаю $w_{2} \leq w_{1}$.

Если $w_{1}=w_{2}$, то, полагая $u_{1}=u-w_{1}, v_{1}=v-w_{1}$, найдем

$$
\operatorname{DiLog} \mathrm{A}=\int_{u_{1}}^{v_{1}} \ln s d s / s=\left.0.5 \cdot \ln ^{2} s\right|_{u_{1}} ^{v_{1}}=0.5 \ln ^{2}\left(v-w_{1}\right)-0.5 \ln ^{2}\left(u-w_{1}\right) \text {. }
$$

Пусть $w_{2}<w_{1}$. Интегрируя по частям, получим

$$
\operatorname{DiLog} \mathrm{A}=\left.\ln \left(t-w_{2}\right) \cdot \ln \frac{t-w_{1}}{w_{1}-w_{2}}\right|_{u} ^{v}+I_{2}, \quad I_{2}=-\int_{u}^{v} \ln \frac{t-w_{1}}{w_{1}-w_{2}} \cdot \frac{d t}{t-w_{2}} .
$$


Делая замену переменной $s=\frac{t-w_{2}}{w_{1}-w_{2}}$, перепишем интеграл $I_{2}$ в виде

$$
I_{2}=-\int_{u_{1}}^{v_{1}} \ln (s-1) d s / s
$$

где $u_{1}=\frac{u-w_{2}}{w_{1}-w_{2}}$ и $v_{1}=\frac{v-w_{2}}{w_{1}-w_{2}}$. Преобразуем подынтегральную функцию: $\ln (s-1)=\ln s+\ln (1-1 / s)$, тогда интеграл (15) сведется к сумме двух интегралов. Значение первого из них мы уже знаем, оно дается формулой (14) со знаком «-». Второй интеграл перепишем в виде

$$
I_{3}=-\int_{u_{1}}^{v_{1}} \ln (1-1 / s) d s / s=-\int_{u_{2}}^{v_{2}} \ln (1-x) d x / x, \quad u_{2}=1 / v_{1}, v_{2}=1 / u_{1} .
$$

Заметим, что $0<u_{2}=\frac{w_{1}-w_{2}}{v-w_{2}}<v_{2}=\frac{w_{1}-w_{2}}{u-w_{2}}<1$. Если $v_{2} \leq 0.5$, то интеграл $I_{3}$ вычисляется непосредственно через разложение в ряд:

$$
I_{3}=\int_{u_{2}}^{v_{2}} \sum_{i>0} \frac{x^{i-1}}{i} d x=\sum_{i>0} \frac{v_{2}^{i}-u_{2}^{i}}{i^{2}} .
$$

Из сделанного выше замечания о том, что $x^{J}=0$, если $x$ - число типа double, $|x| \leq 0.5$, а $J \geq \operatorname{Jmax}=1075$, следует, что элементы ряда (16) достаточно вычислять лишь для значений $i \leq \mathrm{Jmax}$.

Пусть $\mho_{2}>0.5$. Тогда

$$
I_{3}=-\int_{u_{2}}^{0.5} \ln (1-x) d x / x-\int_{0.5}^{v_{2}} \ln (1-x) d x / x,
$$

где первый интеграл считается через разложение в ряд (16) с $i=1, \ldots$, Jmax. Второй интеграл (обозначим его через $I_{4}$ ) переписывается в виде

$$
\begin{aligned}
I_{4}=-\int_{0.5}^{v_{2}} \ln (1-x) d x / x & =\left.\ln (1-x) \cdot \ln x\right|_{v_{2}} ^{0.5}-\int_{0.5}^{v_{2}} \ln x d x /(1-x)= \\
& =\left.\ln (1-x) \cdot \ln x\right|_{v_{2}} ^{0.5}-\int_{1-v_{2}}^{0.5} \ln (1-x) d x / x
\end{aligned}
$$


Последний интеграл вычисляется с помощью разложения в ряд:

$$
I_{5}=-\int_{1-v_{2}}^{0.5} \ln (1-x) d x / x=\int_{1-v_{2}}^{0.5} \sum_{i>0} \frac{x^{i-1}}{i} d x=\sum_{i>0} \frac{0.5^{i}-\left(1-v_{2}\right)^{i}}{i^{2}} .
$$

В последней сумме также достаточно просуммировать лишь первые Jmax членов ряда.

На этом описание метода вычисления интеграла DiLogA закончено.

Переходя к случаю (2), прибавим и вычтем из дилогарифма интеграл

$$
\int_{u}^{v} \ln \left(w_{1}-w_{2}\right) d t /\left(t-w_{1}\right)=\left.\ln \left(w_{1}-w_{2}\right) \ln \left(w_{1}-t\right)\right|_{u} ^{v} .
$$

Тогда

$$
\operatorname{DiLogB}=\left.\ln \left(w_{1}-w_{2}\right) \ln \left(w_{1}-t\right)\right|_{u} ^{v}+I_{1}^{\prime}, \quad I_{1}^{\prime}=\int_{u}^{v} \ln \frac{t-w_{2}}{w_{1}-w_{2}} d t /\left(t-w_{1}\right) .
$$

Сделаем в $I_{1}^{\prime}$ замену переменной $x=\frac{t-w_{2}}{w_{1}-w_{2}}$. Тогда

$$
I_{1}^{\prime}=-\int_{u_{1}}^{v_{1}} \ln x d x /(1-x), \quad \text { где } u_{1}=\frac{u-w_{2}}{w_{1}-w_{2}}, \quad v_{1}=\frac{v-w_{2}}{w_{1}-w_{2}} .
$$

Заметим, что $0<u_{1}<v_{1}<1$. Если $v_{1} \leq 0.5$, то проинтегрируем $I_{1}^{\prime}$ по частям и сведем задачу к случаю, аналогичному (16):

$$
I_{1}^{\prime}=\left.\ln x \cdot \ln (1-x)\right|_{u_{1}} ^{v_{1}}-\int_{u_{1}}^{v_{1}} \ln (1-x) d x / x=\left.\ln x \cdot \ln (1-x)\right|_{u_{1}} ^{v_{1}}+\sum_{i>0} \frac{v_{1}^{i}-u_{1}^{i}}{i^{2}}
$$

В последнем ряде достаточно просуммировать лишь первые Jmax членов ряда.

Если $v_{1}>0.5$, то применим аналогичный прием, заключающийся в разбиении отрезка интегрирования на два подотрезка:

$$
I_{1}^{\prime}=\left.\ln x \cdot \ln (1-x)\right|_{u_{1}} ^{v_{1}}-\int_{u_{1}}^{0.5} \ln (1-x) d x / x-\int_{0.5}^{v_{1}} \ln (1-x) d x / x .
$$


Первый из интегралов справа считается так, как мы только что показали. Для второго интеграла (обозначим его через $I_{2}^{\prime}$ ) используем цепочку преобразований:

$$
\begin{aligned}
I_{2}^{\prime}=-\int_{0.5}^{v_{1}} \ln (1-x) d x / x & =\left.\ln (1-x) \cdot \ln x\right|_{v_{1}} ^{0.5}-\int_{0.5}^{v_{1}} \ln x d x /(1-x)= \\
& =\left.\ln (1-x) \cdot \ln x\right|_{v_{1}} ^{0.5}-\int_{1-v_{1}}^{0.5} \ln (1-x) d x / x .
\end{aligned}
$$

Последний интеграл вычисляется с помощью разложения в ряд:

$$
I_{3}^{\prime}=-\int_{1-v_{1}}^{0.5} \ln (1-x) d x / x=\int_{1-v_{1}}^{0.5} \sum_{i>0} \frac{x^{i-1}}{i} d x=\sum_{i>0} \frac{0.5^{i}-\left(1-v_{1}\right)^{i}}{i^{2}} .
$$

В последней сумме достаточно просуммировать лишь первые Jmax ee членов.

На этом описание метода вычисления интеграла DiLogB закончено.

Для дальнейшего будет удобно определить интеграл DiLog, coвпадающий с интегралом DiLogA в случае (1) и с интегалом DiLogB в случае (2).

\section{3. Алгоритм вычисления значений $\boldsymbol{J}_{2,2}$ и $\boldsymbol{J}_{2,1}$}

Интегралы

$$
J_{2,2}\left(u, v, w, w_{2}\right)=J_{2,2}[i]=\int_{u}^{v}(t-w)^{i} \cdot \ln \left(t-w_{2}\right) d t
$$

вычисляются с помощью рекуррентной формулы

$$
J_{2,2}[i]=\left.\frac{(t-w)^{i+1}}{i+1} \cdot \ln \left(t-w_{2}\right)\right|_{u} ^{v}-\frac{J_{1,1}[i+1]\left(u, v, w, w_{2}\right)}{i+1}, \quad i=0,1,2, \ldots
$$

Здесь предполагается, что уже заранее заготовлена таблица значений интеграла $J_{1,1}[i]\left(u, v, w, w_{2}\right)$ и таблицы $p_{1}[i], p_{2}[i]$ степеней чисел $u-w$, $v-w$.

Интегралы

$$
J_{2,1}\left(u, v, w, w_{1}, w_{2}\right)=J_{2,1}[i]=\int_{u}^{v}(t-w)^{i} \cdot \ln \left(t-w_{2}\right) d t /\left(t-w_{1}\right)
$$


вычисляются с помощью рекуррентной формулы

$$
J_{2,1}[i]=J_{2,2}[i-1]-\left(w-w_{1}\right) \cdot J_{2,1}[i-1], \quad i=0,1,2, \ldots
$$

Аналогично рассмотренному выше интегралу $J_{1,1}[i]$ эта формула применяется в прямом направлении (т. е. для $i=1,2, \ldots$, Jmax) при $\left|w-w_{1}\right| \leq 1$ и в обратном направлении (т. е. для $i=\mathrm{Jmax}, \mathrm{J} \max -1, \ldots, 1)$ при $\left|w-w_{1}\right|>1$, чтобы не было экспоненциального роста ошибок округления. При $i=\mathrm{Jmax}$ полагаем интеграл равным нулю.

\section{4. Алгоритм вычисления значений $\boldsymbol{J}_{3,2}$ и $\boldsymbol{J}_{3,1}$}

Теперь перейдем к рассмотрению интегралов

$$
J_{3,2}\left(u, v, w, w_{1}, w_{2}\right)=\int_{u}^{v}\left(t_{1}-w\right)^{i} d t_{1} \cdot t_{1} \cdot \int_{w_{1}+w_{2}}^{t_{1}-w_{1}} d t_{2} \cdot \ln \left(t_{2}-w_{2}\right) /\left(t_{2}\left(t_{1}-t_{2}\right)\right)
$$

и

$$
J_{3,1}\left(u, v, w, w_{1}, w_{2}\right)=\int_{u}^{v}\left(t_{1}-w\right)^{i} d t_{1} \cdot \int_{w_{1}+w_{2}}^{t_{1}-w_{1}} d t_{2} \cdot \ln \left(t_{2}-w_{2}\right) /\left(t_{2}\left(t_{1}-t_{2}\right)\right) .
$$

Используя равенство $\frac{t_{1}}{t_{2}\left(t_{1}-t_{2}\right)}=\frac{1}{t_{2}}+\frac{1}{t_{1}-t_{2}}$, запишем интеграл $J_{3,2}\left(u, v, w, w_{1}, w_{2}\right)=J_{3,2}[i]$ в виде суммы двух интегралов

$$
\begin{aligned}
J_{3,2}[i] & =\int_{u}^{v}\left(t_{1}-w\right)^{i} d t_{1} \int_{w_{1}+w_{2}}^{t_{1}-w_{1}} \ln \left(t_{2}-w_{2}\right) d t_{2} / t_{2}+ \\
& +\int_{u}^{v}\left(t_{1}-w\right)^{i} d t_{1} \int_{w_{1}+w_{2}}^{t_{1}-w_{1}} \ln \left(t_{2}-w_{2}\right) d t_{2} /\left(t_{1}-t_{2}\right) .
\end{aligned}
$$

Интегрируя по частям каждый из интегралов в правой части, получим, что $J_{3,2}[i]=A_{1}-B_{1}$, где

$$
\begin{aligned}
& A_{1}=\left.\frac{\left(t_{1}-w\right)^{i+1}}{i+1} \cdot\left(\int_{w_{1}+w_{2}}^{t_{1}-w_{1}} \ln \left(t_{2}-w_{2}\right) d t_{2} / t_{2}+\int_{w_{1}+w_{2}}^{t_{1}-w_{1}} d t_{2} \cdot \frac{\ln \left(t_{2}-w_{2}\right)}{t_{1}-t_{2}}\right)\right|_{t_{1}=u} ^{t_{1}=v}, \\
& B_{1}=\int_{u}^{v} \frac{\left(t_{1}-w\right)^{i+1}}{i+1} \cdot \frac{d}{d t_{1}}\left(\int_{w_{1}+w_{2}}^{t_{1}-w_{1}} \ln \left(t_{2}-w_{2}\right) d t_{2} / t_{2}+\int_{w_{1}+w_{2}}^{t_{1}-w_{1}} \frac{\ln \left(t_{2}-w_{2}\right)}{t_{1}-t_{2}} d t_{2}\right) .
\end{aligned}
$$


Величину $A_{1}$ мы уже умеем вычислять, она есть сумма четырех дилогарифмов с соответствующими коэффициентами:

$$
\begin{aligned}
& A_{1}= \\
& =\frac{p_{2}[i+1]}{i+1}\left(\operatorname{DiLog}\left(w_{1}+w_{2}, v-w_{1}, 0, w_{2}\right)-\operatorname{DiLog}\left(w_{1}+w_{2}, v-w_{1}, v, w_{2}\right)\right)- \\
& -\frac{p_{1}[i+1]}{i+1}\left(\operatorname{DiLog}\left(w_{1}+w_{2}, u-w_{1}, 0, w_{2}\right)-\operatorname{DiLog}\left(w_{1}+w_{2}, u-w_{1}, u, w_{2}\right)\right) .
\end{aligned}
$$

Вычисляя производную в выражении для $B_{1}$, получим

$$
\begin{aligned}
B_{1} & =\int_{u}^{v} \frac{\left(t_{1}-w\right)^{i+1}}{i+1} \ln \left(t_{1}-w_{1}-w_{2}\right)\left(\frac{1}{t_{1}-w_{1}}+\frac{1}{w_{1}}\right)- \\
& -\int_{u}^{v} \frac{\left(t_{1}-w\right)^{i+1}}{i+1} \int_{w_{1}+w_{2}}^{t_{1}-w_{1}} \ln \left(t_{2}-w_{2}\right) \frac{d t_{2}}{\left(t_{1}-t_{2}\right)^{2}} .
\end{aligned}
$$

Внутренний интеграл во втором интеграле в правой части можно вычислить по частям:

$$
\int_{w_{1}+w_{2}}^{t_{1}-w_{1}} \ln \left(t_{2}-w_{2}\right) \frac{d t_{2}}{\left(t_{1}-t_{2}\right)^{2}}=\left.\frac{\ln \left(t_{2}-w_{2}\right)}{t_{1}-t_{2}}\right|_{w_{1}+w_{2}} ^{t_{1}-w_{1}}-\int_{w_{1}+w_{2}}^{t_{1}-w_{1}} \frac{d t_{2}}{\left(t_{2}-w_{2}\right)\left(t_{1}-t_{2}\right)} .
$$

Последний интеграл вычисляется непосредственно:

$$
\begin{gathered}
\int_{w_{1}+w_{2}}^{t_{1}-w_{1}} \frac{d t_{2}}{\left(t_{2}-w_{2}\right)\left(t_{1}-t_{2}\right)}=\int_{w_{1}+w_{2}}^{t_{1}-w_{1}}\left(\frac{1}{t_{2}-w_{2}}+\frac{1}{t_{1}-t_{2}}\right) \frac{d t_{2}}{t_{1}-w_{2}}= \\
=\left.\ln \frac{t_{2}-w_{2}}{t_{1}-t_{2}} \cdot \frac{1}{t_{1}-w_{2}}\right|_{w_{1}+w_{2}} ^{t_{1}-w_{1}} .
\end{gathered}
$$

Собирая все вместе, получаем следующее выражение для $B_{1}$ :

$$
\begin{aligned}
B_{1} & =\int_{u}^{v} \frac{\left(t_{1}-w\right)^{i+1}}{i+1} \ln \left(t_{1}-w_{1}-w_{2}\right)\left(\frac{1}{t_{1}-w_{1}}+\frac{1}{w_{1}}\right) \\
& -\int_{u}^{v} \frac{\left(t_{1}-w\right)^{i+1}}{i+1}\left(\left.\frac{\ln \left(t_{2}-w_{2}\right)}{t_{1}-t_{2}}\right|_{w_{1}+w_{2}} ^{t_{1}-w_{1}}-\left.\ln \frac{t_{2}-w_{2}}{t_{1}-t_{2}} \cdot \frac{1}{t_{1}-w_{2}}\right|_{w_{1}+w_{2}} ^{t_{1}-w_{1}}\right) .
\end{aligned}
$$


После элементарных преобразований находим

$$
\begin{gathered}
B_{1}=\int_{u}^{v} \frac{\left(t_{1}-w\right)^{i+1}}{i+1}\left(\ln \left(t_{1}-w_{1}-w_{2}\right)\left(\frac{1}{t_{1}-w_{1}}+\frac{2}{t_{1}-w_{2}}\right)+\right. \\
\left.\quad+\ln w_{1}\left(\frac{1}{t_{1}-w_{1}-w_{2}}-\frac{2}{t_{1}-w_{2}}\right)\right) d t_{1} .
\end{gathered}
$$

Таким образом, интеграл $B_{1}$ равен комбинации интегралов $J_{2,1}$ и $J_{1,1}$ :

$$
\begin{aligned}
B_{1} & =\frac{1}{i+1} \cdot\left(J_{2,1}[i+1]\left(u, v, w, w_{1}, w_{1}+w_{2}\right)+2 J_{2,1}[i+1]\left(u, v, w, w_{2}, w_{1}+w_{2}\right)\right) \\
& +\frac{1}{i+1} \cdot \ln w_{1} \cdot\left(J_{1,1}[i+1]\left(u, v, w, w_{1}+w_{2}\right)-2 J_{1,1}[i+1]\left(u, v, w, w_{2}\right)\right) .
\end{aligned}
$$

Здесь предполагается, что уже заранее заготовлены таблицы J_11[i] и JJ_11[i] значений интегралов $J_{1,1}[i]\left(u, v, w, w_{1}+w_{2}\right)$ и $J_{1,1}[i]\left(u, v, w, w_{2}\right)$, а также таблицы J_21[i] и JJ_21[i] значений интегралов $J_{2,1}[i]\left(u, v, w, w_{1}, w_{1}+w_{2}\right)$ и $J_{2,1}[i]\left(u, v, w, w_{2}, w_{1}+w_{2}\right)$. Для последних двух таблиц требуется также заранее заготовленная таблица значений интеграла $J_{2,2}[i]\left(u, v, w, w_{1}+w_{2}\right)$.

Интегралы $J_{3,1}[i]$ вычисляются с помощью рекуррентной формулы

$$
J_{3,1}[i]=J_{3,2}[i-1]-w \cdot J_{3,1}[i-1], \quad i=1,2, \ldots, \text { Jmax, }
$$

которая применяется в обратном направлении (т. е. при $i=\operatorname{Jmax}, \operatorname{Jmax}-$ $-1, \ldots, 1)$, поскольку предполагается, что $w>1$, и нужно не допускать экспоненциального роста ошибок округления. При $i=\operatorname{Jmax}$ полагаем интеграл равным нулю.

\section{5. Алгоритм вычисления значений $\boldsymbol{F}_{1}, \boldsymbol{F}_{2}$ и $\boldsymbol{F}_{3}$}

В данном разделе мы рассмотрим вопрос вычисления следующих трех интегралов

(1) $F_{1}=\int_{A}^{B} \rho_{k}\left(\left(1-\mu_{1}\right) / \alpha\right) d \mu_{1} / \mu_{1}$;

(2) $F_{2}=\int_{A}^{B} \rho_{k}\left(\left(1-\mu_{1}\right) / \alpha\right) \cdot \ln \left(\left(\mu_{1}-\gamma_{1}\right) / \gamma_{1}\right) d \mu_{1} / \mu_{1}$;

(3) $F_{3}=\int_{A}^{B} \rho_{k}\left(\left(1-\mu_{1}\right) / \alpha\right) d \mu_{1} \int_{\gamma_{1}+\gamma_{2}}^{\mu_{1}-\gamma_{1}} \ln \left(\left(\mu_{2}-\gamma_{2}\right) / \gamma_{2}\right) d \mu_{2} /\left(\mu_{2}\left(\mu_{1}-\mu_{2}\right)\right)$.

Во всех трех случаях предполагается, что $\left(1-\mu_{1}\right) / \alpha=k-0.5-x$, где $x \in[-0.5,0.5]$, если $\mu_{1} \in[A, B]$.

Выразим $x$ через $\mu_{1}$ :

$$
x=k-0.5-\frac{1-\mu_{1}}{\alpha}=\frac{\mu_{1}}{\alpha}-\frac{\lambda_{k}}{\alpha}, \quad \lambda_{k}=1-\alpha(k-0.5) .
$$


Пусть

$$
u=A / \alpha, v=B / \alpha, w=\lambda_{k} / \alpha, w_{1}=\gamma_{1} / \alpha \text { и } w_{2}=\gamma_{2} / \alpha .
$$

Разлагая функцию Дикмана в ряд Тейлора (7) и меняя порядок суммирования и интегрирования, мы сводим задачу вычисления интегралов (1)-(3) к задаче вычисления рассмотренных ранее интегралов:

$$
\begin{aligned}
& F_{1}=\sum_{i \geq 0} c_{k, i} \cdot J_{1,1}(u, v, w, 0), \\
& F_{2}=\sum_{i \geq 0} c_{k, i}\left(J_{2,1}\left(u, v, w, 0, w_{1}\right)-\ln w_{1} \cdot J_{1,1}(u, v, w, 0)\right), \\
& F_{3}=\sum_{i \geq 0} c_{k, i}\left(J_{3,1}\left(u, v, w, w_{1}, w_{2}\right)-\ln w_{2} \int_{u}^{t_{1}-w_{1}}\left(t_{1}-w\right)^{i} d t_{1} \int_{w_{1}+w_{2}}^{t_{2}} \frac{d t_{2}}{t_{2}\left(t_{1}-t_{2}\right)}\right) .
\end{aligned}
$$

Интеграл внутри суммы правой части последнего равенства равен

$$
\begin{gathered}
\left.\int_{u}^{v}\left(t_{1}-w\right)^{i} \frac{d t_{1}}{t_{1}} \cdot \ln \frac{t_{2}}{t_{1}-t_{2}}\right|_{w_{1}+w_{2}} ^{t_{1}-w_{1}}= \\
=\int_{u}^{v}\left(t_{1}-w\right)^{i} \frac{d t_{1}}{t_{1}} \cdot\left(\ln \frac{t_{1}-w_{1}}{w_{1}}+\ln \frac{t_{1}-w_{1}-w_{2}}{w_{1}+w_{2}}\right)= \\
=J_{2,1}\left(u, v, w, 0, w_{1}\right)-\ln \left(w_{1}^{2}+w_{1} w_{2}\right) J_{1,1}(u, v, w, 0)+J_{2,1}\left(u, v, w, 0, w_{1}+w_{2}\right) .
\end{gathered}
$$

Ниже, если надо подчеркнуть зависимость от параметров, будем использовать обозначение $F_{i}(k, \alpha, A, B), i=1,2,3$.

\section{6. Алгоритм вычисления значений $\boldsymbol{G}_{1}, \boldsymbol{G}_{2}$ и $\boldsymbol{G}_{3}$}

Согласно формуле (5) функция $G_{1}\left(\alpha, \beta_{1}, \beta_{2}\right)$ равна интегралу

$$
G_{1}\left(\alpha, \beta_{1}, \beta_{2}\right)=\int_{\beta_{1}}^{\beta_{2}} \rho((1-\mu) / \alpha) d \mu / \mu .
$$

Пусть $\mu_{k}=1-k \alpha$ и $\lambda_{k}=1-(k-0.5) \alpha, k=0,1, \ldots$ Тогда $\lceil(1-$ $-\mu) / \alpha\rceil=k$ при $\mu \in\left[\mu_{k}, \mu_{k-1}\right)$. Следовательно,

$$
\rho\left(\frac{1-\mu}{\alpha}\right)=\rho_{k}\left(\frac{1-\mu}{\alpha}\right)=\sum_{i \geq 0} c_{k, i} x^{i}, \quad x=k-0.5-\frac{1-\mu}{\alpha}=\frac{\mu}{\alpha}-\frac{\lambda_{k}}{\alpha} .
$$


Пусть

$$
k_{\gamma}=\max \left(k: \mu_{k}>\gamma\right)=\left\lceil\frac{1-\gamma}{\alpha}\right\rceil-1, \quad K_{\gamma}=\min \left(k: \mu_{k}<\gamma\right)=\left\lfloor\frac{1-\gamma}{\alpha}\right\rfloor+1 .
$$

Имеем два случая.

(1) На интервале $\left(\frac{1-\beta_{2}}{\alpha}, \frac{1-\beta_{1}}{\alpha}\right)$ нет целых точек. Тогда $k_{\beta_{1}}=K_{\beta_{2}}-1$ и $G_{1}\left(\alpha, \beta_{1}, \beta_{2}\right)=\int_{\beta_{1}}^{\beta_{2}} \rho_{K}((1-\mu) / \alpha) d \mu / \mu=F_{1}\left(K, \alpha, \beta_{1}, \beta_{2}\right), \quad K=K_{\beta_{2}}$.

(2) На интервале $\left(\frac{1-\beta_{2}}{\alpha}, \frac{1-\beta_{1}}{\alpha}\right)$ есть целые точки. Тогда $k_{\beta_{1}} \geq K_{\beta_{2}}$ и

$$
\begin{gathered}
G_{1}\left(\alpha, \beta_{1}, \beta_{2}\right)=\int_{\beta_{1}}^{\mu_{\beta_{\beta_{1}}}} \rho_{k_{\beta_{1}}+1}\left(\frac{1-\mu}{\alpha}\right) d \mu / \mu+\int_{\mu_{\beta_{\beta_{2}}}}^{\beta_{2}} \rho_{K_{\beta_{2}}}\left(\frac{1-\mu}{\alpha}\right) d \mu / \mu+ \\
+\sum_{K_{\beta_{2}}+1 \leq l \leq k_{\beta_{1}}} \int_{\mu_{l}}^{\mu_{l-1}} \rho_{l}\left(\frac{1-\mu}{\alpha}\right) d \mu / \mu=F_{1}\left(k+1, \alpha, \beta_{1}, \mu_{k}\right)+F_{1}\left(K, \alpha, \mu_{K}, \beta_{2}\right)+ \\
+\sum_{K+1 \leq l \leq k} F_{1}\left(l, \alpha, \mu_{l}, \mu_{l-1}\right), \quad k=k_{\beta_{1}}, K=K_{\beta_{2}} .
\end{gathered}
$$

Для функции $G_{2}\left(\alpha, \beta_{1}, \beta_{2}\right)$ воспользуемся формулой (1):

$$
G_{2}\left(\alpha, \beta_{1}, \beta_{2}\right)=\sum_{\gamma \in\left\{\beta_{1}, \beta_{2}\right\}} e(\gamma) J(\gamma), \quad J(\gamma)=\int_{\beta_{1}+\gamma}^{\beta_{2}+\gamma} \rho\left(\frac{1-\mu}{\alpha}\right) \frac{d \mu}{\mu} \cdot \ln \frac{\mu-\gamma}{\gamma} .
$$

Аналогично предыдущему имеем два случая.

(1) На интервале $\left(\frac{1-\beta_{2}-\gamma}{\alpha}, \frac{1-\beta_{1}-\gamma}{\alpha}\right)$ нет целых точек. Тогда $k_{\beta_{1}+\gamma}=$ $=K_{\beta_{2}+\gamma}-1$ и

$$
J(\gamma)=\int_{\beta_{1}+\gamma}^{\beta_{2}+\gamma} \rho_{K}\left(\frac{1-\mu}{\alpha}\right) \cdot \ln \left(\frac{\mu-\gamma}{\gamma}\right) d \mu / \mu=F_{2}\left(K, \alpha, \beta_{1}+\gamma, \beta_{2}+\gamma\right), \quad K=K_{\beta_{2}+\gamma} .
$$


$\geq K_{\beta_{2}+\gamma}$ и

(2) На интервале $\left(\frac{1-\beta_{2}-\gamma}{\alpha}, \frac{1-\beta_{1}-\gamma}{\alpha}\right)$ есть целые точки. Тогда $k_{\beta_{1}+\gamma} \geq$

$$
\begin{gathered}
J(\gamma)=\int_{\beta_{1}+\gamma}^{\mu_{k_{1}+\gamma}} \rho_{k_{\beta_{1}+\gamma}+1}\left(\frac{1-\mu}{\alpha}\right) \cdot \ln \left(\frac{\mu-\gamma}{\gamma}\right) d \mu / \mu+ \\
+\int_{\mu_{\beta_{\beta_{2}+\gamma}}^{\beta_{2}+\gamma} \rho_{K_{\beta_{2}+\gamma}}\left(\frac{1-\mu}{\alpha}\right) \cdot \ln \left(\frac{\mu-\gamma}{\gamma}\right) d \mu / \mu+} \\
+\sum_{K_{\beta_{2}+\gamma}+1 \leq l \leq k_{\beta_{1}+\gamma}} \int_{\mu_{l}}^{\mu_{l-1}} \rho_{l}\left(\frac{1-\mu}{\alpha}\right) \cdot \ln \left(\frac{\mu-\gamma}{\gamma}\right) d \mu / \mu= \\
=F_{2}\left(k+1, \alpha, \beta_{1}+\gamma, \mu_{k}\right)+F_{2}\left(K, \alpha, \mu_{K}, \beta_{2}+\gamma\right)+\sum_{K+1 \leq l \leq k} F_{2}\left(l, \alpha, \mu_{l}, \mu_{l-1}\right), \\
k=k_{\beta_{1}+\gamma}, \quad K=K_{\beta_{2}+\gamma} .
\end{gathered}
$$

Для функции $G_{3}\left(\alpha, \beta_{1}, \beta_{2}\right)$ воспользуемся формулой (2):

$$
\begin{gathered}
G_{3}\left(\alpha, \beta_{1}, \beta_{2}\right)=\frac{1}{3} \sum_{\gamma_{1}, \gamma_{2} \in\left\{\beta_{1}, \beta_{2}\right\}} e\left(\gamma_{1}\right) e\left(\gamma_{2}\right) J\left(\gamma_{1}, \gamma_{2}\right), \\
J\left(\gamma_{1}, \gamma_{2}\right)=\int_{\beta_{1}+\gamma_{1}+\gamma_{2}}^{\beta_{2}+\gamma_{1}+\gamma_{2}} \rho\left(\frac{1-\mu_{1}}{\alpha}\right) d \mu_{1} \times \int_{\gamma_{1}+\gamma_{2}}^{\mu_{1}-\gamma_{1}} \frac{d \mu_{2}}{\left(\mu_{1}-\mu_{2}\right) \mu_{2}} \cdot \ln \frac{\mu_{2}-\gamma_{2}}{\gamma_{2}} .
\end{gathered}
$$

Снова рассмотрим два случая.

(1) На интервале $\left(\frac{1-\beta_{2}-\gamma_{1}-\gamma_{2}}{\alpha}, \frac{1-\beta_{1}-\gamma_{1}-\gamma_{2}}{\alpha}\right)$ нет целых точек. Тогда $k_{\beta_{1}+\gamma_{1}+\gamma_{2}}=K_{\beta_{2}+\gamma_{1}+\gamma_{2}}-1$ и

$$
\begin{aligned}
J\left(\gamma_{1}, \gamma_{2}\right) & =\int_{\beta_{1}+\gamma_{1}+\gamma_{2}}^{\beta_{2}+\gamma_{1}+\gamma_{2}} \rho_{K}\left(\frac{1-\mu_{1}}{\alpha}\right) d \mu_{1} \int_{\gamma_{1}+\gamma_{2}}^{\mu_{1}-\gamma_{1}} \ln \left(\frac{\mu_{2}-\gamma_{2}}{\gamma_{2}}\right) \frac{d \mu_{2}}{\mu_{2}\left(\mu_{1}-\mu_{2}\right)}= \\
& =F_{3}\left(K, \alpha, \beta_{1}+\gamma_{1}+\gamma_{2}, \beta_{2}+\gamma_{1}+\gamma_{2}\right), \quad K=K_{\beta_{2}+\gamma_{1}+\gamma_{2}} .
\end{aligned}
$$


(2) На интервале $\left(\frac{1-\beta_{2}-\gamma_{1}-\gamma_{2}}{\alpha}, \frac{1-\beta_{1}-\gamma_{1}-\gamma_{2}}{\alpha}\right)$ есть целые точки. Тогда $k_{\beta_{1}+\gamma_{1}+\gamma_{2}} \geq K_{\beta_{2}+\gamma_{1}+\gamma_{2}}$ и

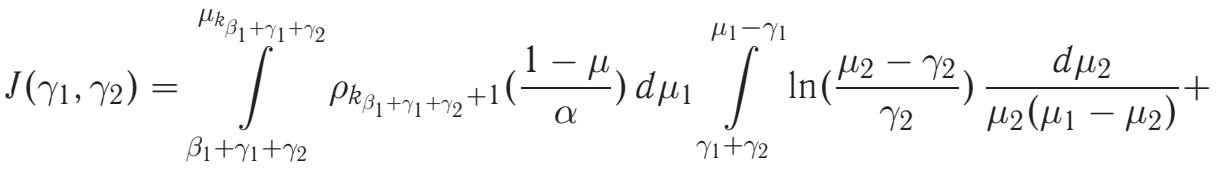

$$
\begin{aligned}
& +\int_{\mu_{K_{\beta_{2}}+\gamma_{1}+\gamma_{2}}}^{\beta_{2}+\gamma_{1}+\gamma_{2}} \rho_{K_{\beta_{2}+\gamma_{1}+\gamma_{2}}}\left(\frac{1-\mu}{\alpha}\right) d \mu_{1} \int_{\gamma_{1}+\gamma_{2}}^{\mu_{1}-\gamma_{1}} \ln \left(\frac{\mu_{2}-\gamma_{2}}{\gamma_{2}}\right) \frac{d \mu_{2}}{\mu_{2}\left(\mu_{1}-\mu_{2}\right)}+ \\
& +\sum_{K_{\beta_{2}+\gamma_{1}+\gamma_{2}}+1 \leq l \leq k_{\beta_{1}+\gamma_{1}+\gamma_{2}}} \int_{\mu_{l}}^{\mu_{l}-1} \rho_{l}\left(\frac{1-\mu}{\alpha}\right) d \mu_{1} \int_{\gamma_{1}+\gamma_{2}}^{\mu_{1}-\gamma_{1}} \ln \left(\frac{\mu_{2}-\gamma_{2}}{\gamma_{2}}\right) \frac{d \mu_{2}}{\mu_{2}\left(\mu_{1}-\mu_{2}\right)}= \\
& =F_{3}\left(k+1, \alpha, \beta_{1}+\gamma_{1}+\gamma_{2}, \mu_{k}\right)+F_{3}\left(K, \alpha, \mu_{K}, \beta_{2}+\gamma_{1}+\gamma_{2}\right)+ \\
& +\sum_{K+1 \leq l \leq k} F_{3}\left(l, \alpha, \mu_{l}, \mu_{l-1}\right), \quad k=k_{\beta_{1}+\gamma_{1}+\gamma_{2}}, K=K_{\beta_{2}+\gamma_{1}+\gamma_{2}} \text {. }
\end{aligned}
$$

\section{6. Трудоемкость вычисления значений функций $\boldsymbol{G}_{2}$ и $\boldsymbol{G}_{3}$}

Как следует из предложенного метода, значения функций $G_{t}, t=2,3$, являются суммами не более $K=\left\lceil\frac{\beta_{2}}{\alpha}\right\rceil+2$ значений функций $F_{t}, t=2,3$, поэтому для вычисления значений $G_{t}$ с абсолютной погрешностью $\delta$ достаточно вычислить все встречающиеся значения $F_{t}$ с абсолютной погрешностью $\delta / K$.

В свою очередь, функции $F_{t}=F_{t}(k)$ являются суммами рядов, элементы которых (обозначим их через $a_{k, i}$ ) являются линейными комбинациями интегралов типа $J$. С ростом $i$ элементы $a_{k, i}$ убывают по абсолютной величине со скоростью геометрической прогрессии равномерно по всем $k \leq K$. Поэтому, чтобы вычислить сумму такого ряда с абсолютной погрешностью $\delta_{1}$, достаточно учесть лишь $O\left(\ln \delta_{1}\right)$ первых членов ряда.

Это показывает, что для вычисления значений функций $G_{t}$ с фиксированной абсолютной погрешностью достаточно вычислить $O(K \ln K)$ интегралов типа $J$.

Интегралы типа $J$ зависят от параметров $u, v, w, w_{1}$ и $\varpi_{2}$. Из описания метода следует, что параметры $u, v$ и ш этих интегралов могут пробегать не более $K$ возможных значений, а параметры $u-w, v-w, w_{1}$ и $w_{2}$ не более $O(1)$ возможных значений. 
Вычисление всех интегралов типа $J_{1,1}$ требует $O(K \ln K)$ операций сложения и умножения с плавающей точкой, $O(K)$ операций деления (необходимых для обращения чисел вида $\left.-w_{1}\right)$ и $O(1)$ операций вычисления логарифма (требуемых в тех случаях, когда $\mid$ - $w_{1} \mid \leq 1$ ). Обозначим эту трудоемкость через $T_{1,1}$.

Интегралы типа $J_{2,2}$ вычисляются через интегралы типа $J_{1,1}$, но требуют также вычисления логарифмов чисел $u-w_{2}$ и $v-w_{2}$. По этой причине трудоемкость $T_{2,2}$ вычисления этих интегралов является величиной $O\left(T_{1,1}+K T_{\log }\right)$, где $T_{\log }$ - трудоемкость вычисления одного логарифма.

Трудоемкость $T_{2,1}$ вычисления интегралов $J_{2,1}$ является величиной такого же порядка. Аналогичное, как легко видеть, имеет место и для интегралов $J_{3,2}$ и $J_{3,1}$.

Подводя итог, получаем, что для вычисления функций $G_{t}, t=2,3$, с фиксированной абсолютной погрешностью нужно провести $O(K \ln K)$ операций сложения и умножения с плавающей точкой, $O(K)$ операций деления и $O(K)$ операций вычисления логарифма.

\section{7. Результаты вычислительных экспериментов}

Существуют известные универсальные методы вычисления кратных интегралов. Для экспериментов и последующего сравнительного анализа нами были реализованы: метод Монте-Карло, метод Симпсона, метод Коробова [1], использующий параллелепипедальные сетки специального вида, а также методы, приведенные выше. Все программные коды написаны на языке C.

При сравнении методов нас будут интересовать две естественные характеристики: скорость и точность вычисления многомерных интегралов с помощью каждого из методов. Заметим, что точность методов, основанных на квадратурных формулах (это методы Монте-Карло, Симпсона и Коробова), надо задавать отдельно, и чем больше задаваемая точность, тем больше трудоемкость подсчета интеграла. Что же касается методов, предложенных в настоящей работе, то здесь ситуация иная. Нам не надо задавать параметр точности, поскольку значение интеграла во всех случаях вычисляется почти с максимальной точностью, задаваемой разрядностью типа double (около 15 десятичных знаков).

Для значений $\alpha=0.09, \beta_{1}=0.19, \beta_{2}=0.29$ были вычислены значения интегралов $G_{2}\left(\alpha, \beta_{1}, \beta_{2}\right)$ и $G_{3}\left(\alpha, \beta_{1}, \beta_{2}\right)$ с точностью до $3,4,5$ и 6 знаков мантиссы перечисленными методами. В таблице 1 приведены значения времени вычислений в секундах. В последнем столбце приведены времена 
вычислений (с точностью порядка $10^{-15}$ ) методом, основанным на использовании выведенных в статье формул.

Таблица 1. Время вычисления значений функций $G_{m}, m=2,3$

\begin{tabular}{|c|c|c|c|c|}
\hline & $\begin{array}{l}\text { Монте-Карло } \\
\text { e-3...e-6 }\end{array}$ & $\begin{array}{l}\text { Симпсон } \\
\text { e-3...e-6 }\end{array}$ & $\begin{array}{l}\text { Коробов } \\
\text { e-3...e-6 }\end{array}$ & Наш алгоритм \\
\hline \multirow{4}{*}{$G_{2}$} & $e-3: \quad 1.81 e-2$ & $e-3: 8.63 e-4$ & $e-3: 5.56 e-5$ & \multirow{4}{*}{$5.49 e-4$} \\
\hline & $1.64 e-1$ & $8.63 e-4$ & $5.55 e-5$ & \\
\hline & $2.40 e+0$ & $8.64 e-4$ & $1.61 e-4$ & \\
\hline & $9.59 e+0$ & $3.34 e-3$ & $1.61 e-4$ & \\
\hline \multirow{4}{*}{$G_{3}$} & $6.20 e-2$ & $2.56 e-2$ & $e-3: 2.83 e-4$ & \multirow{4}{*}{$4.56 e-3$} \\
\hline & $2.16 e+0$ & $2.56 e-2$ & $e-4: \quad 5.25 e-4$ & \\
\hline & $3.39 e+0$ & $2.56 e-2$ & $9.72 e-4$ & \\
\hline & $2.16 e+2$ & $2.58 e-2$ & $1.12 e-2$ & \\
\hline
\end{tabular}

Как видно из приведенных результатов, при уровне точности от 3 до 5 знаков мантиссы предложенный в настоящей работе алгоритм по скорости вычислений уступает только методу Коробова и существенно превосходит остальные рассмотренные методы. При 6 и более знаках мантиссы предложенный алгоритм становится быстрее и метода Коробова, и чем больше заданная точность вычислений, тем больше превосходство этого алгоритма по отношению к известным методам, основанным на применении квадратурных формул.

Все вычисления проводились на персональном компьютере НP хw6400 Workstation с процессором Intel Xeon $3 \mathrm{GHz}$ под операционной системой Windows XP x64.

\section{Список литературы}

1. Коробов Н. М. Теоретико-числовые методы в приближенном анализе. М.: МЦНМО, 2004.

2. Bach E. The asymptotic behavior of a function occurring in the theory of primes // J. Indian Math. Soc. - 1951. - V. 15. - P. 25-32.

3. Bach E., Peralta R. Asymptotic semismoothness probabilities // Math. of Comput. - 1996. - V. 65. - P. 1701-1715.

4. Ekkelkamp W.H. On the amount of sieving in factorization methods // Ph. D. thesis, The Univ. of Leiden. - 2010.

5. Knuth D. E., Pardo L. T. Analysis of a simple factorization algorithm // Theor. Comput. Sci. - 1976. - V. 3. - P. 321-348.

6. Lambert $R$. Computational aspects of discrete logarithms // Ph. D. thesis., The Univ. of Waterloo. - 1996. 